\title{
Efficient scattering angle filtering for Full waveform inversion
}

Tariq Alkhalifah, KAUST

\section{SUMMARY}

Controlling the scattering angles between the state and the adjoint variables for the energy admitted into an inversion gradient or an image can help improve these functions for objectives in full waveform inversion (FWI) or seismic imaging. However, the access of the scattering angle information usually requires an axis extension that could be costly, especially in 3D. For the purpose of a scattering angle filter, I develop techniques that utilize the mapping nature (no domain extension) of the filter for constant-velocity background models to interpolate between such filtered gradients using the actual velocity. The concept has well known roots in the application of phase-shift-plus-interpolation utilized commonly in the downward continuation process. If the difference between the minimum and maximum velocity of the background medium is large, we obtain filtered gradients corresponding to more constant velocity backgrounds and use linear interpolation between such velocities. The accuracy of this approximation for the Marmousi model gradient demonstrates the effectiveness of the approach.

\section{INTRODUCTION}

The linear component of waveform inversion, the gradient, reflected in an imaging-like process, involves correlating two wavefields. In the classical full waveform inversion (FWI) implementation, one wavefield corresponds to the source, referred to as the state variable, and the other corresponds to the receiver wavefield for that source, referred to as the adjoint variable (Sirgue and Pratt, 2004). The resolution of the resulting gradient, governed by diffraction tomography principles, is controlled by, among other things, the angle between the two interacting wavefields. As the process of imaging is meant to establish the form of the continuation between the two wavefields, whether such continuation is based on a reflection point, or a mere transmission, the scattering angle can readily define for us the nature of the wavefield continuation. As transmissions are a result of wavefields continuing in their path (like direct and diving waves), the scattering angle between the wavefield is almost 180 degrees. On the other hand, reflections admit much smaller scattering angles starting from zero for the majority of zero offset energy to around 80 for large offsets. Conversely, the resolution of the resulting image or gradient is governed by the scattering angle, as it is proportional it's cosine. An image or a gradient can be filtered by applying constraints to the scattering angles contributing to the image.

Controlling the scattering angle content in an image has been administrated indirectly through image filtering methods. In imaging, our goal is to obtain a sharp high resolution image. A goal that tends to be challenged by the imaging transmission components of the wavefield, like diving waves. These components also are present when the Green's functions include reflections, resulting in low frequency artifacts in the image, like those we see in models containing salt bodies (Yoon et al., 2004). Zhang and Sun (2008) suggested a Laplacian filter to mitigate such noise, which is equivalent to multiplying the image contribution by a filter given by the cosine squared of the scattering angle. Khalil et al. (2013) recognized that the true control of such noise is directly attainable through accessing the scattering angle information, so they suggested an extended image using a normalized time lag to efficiently filter out the highscattering angle. Alkhalifah (2015b) used this approach to develop an FWI gradient filtering process that tends to filter out the low scattering angle in the early stages of FWI. An anisotropic media version of the scheme allowed for selective scattering angle filtering to the multi parameter gradient with options that could help mitigate the tradeoff between these parameters (Alkhalifah, 2015a).

In FWI, the process of extending the gradient with an additional dimension at every iteration can be overwhelming, especially in 3D. The extension is needed to properly represent the velocity dependent nature of the smoothing operator given by the scattering angle filter. In the initial iterations, we keep only high scattering angle energy in the gradient, which correspond to mainly transmissions, and results in low wavenumber gradients. For a constant velocity background, the filter is stationary and no extension is needed to apply the filter. In FWI, the purpose is to admit locally higher wavenumbers as we iterate, so the background velocity always tends to have lower wavenumber, and the admission of such wavenumbers is optimally executed through the scattering angle continuation Alkhalifah (2015b). In this abstract, I develop an approach that utilizes the stationary nature of the homogeneous background medium filter to implement a couple of such filters independently and use the velocity to interpolate the results. The idea has it's roots in the phase-shift-plus-interpolation downward continuation developed by (Gazdag and Sguazzero, 1984).

\section{FILTERING THE IMAGE OR GRADIENT}

The gradient in FWI is obtained usually using the adjoint state method, which requires correlating the state wavefield with the adjoint wavefield to acquire the scattering potential at every point of the model. A similar 
approach has been utilized for years in the linear process of imaging using reverse time migration. In imaging the angle between the two wavefields are often utilized for velocity quality control purposes. However, accessing such information required extending the domain of imaging using space or time lags (Sava and Fomel, 2006), which tends to be costly. Using the scattering angle information we can filter the image to remove unwanted low frequency noise (Khalil et al., 2013). We can also filter the gradient to admit more linear components to the velocity model (Alkhalifah, 2015b).

Using the normalized time-lag representation (Khalil et al., 2013), the process of image (or gradient), $R(\mathbf{x})$, extension in the frequency domain is given by the following formula

$$
R(\mathbf{x}, \zeta)=R(\mathbf{x}) e^{-2 i \omega \frac{\zeta}{v(\mathbf{x})}}
$$

where $v$ is the velocity defined using the space variable vector $\mathbf{x}, \zeta$ is the time normalized extension parameter, and omega is the angular frequency. An inherent feature of this modified time-lag (distance units) representation is that the relationship of the scattering angle to the wavenumber of the gradient is free of a velocity (space) dependency. In fact, the scattering angle, $\theta$, is then given by the following formula:

$$
\cos ^{2} \frac{\theta}{2}=\frac{|\mathbf{k}|^{2}}{k_{\zeta}^{2}}
$$

where $\mathbf{k}$ is the wavenumber vector and $k_{\zeta}$ is the wavenumber (Fourier transform) corresponding to $\zeta$. A fourdimensional Fourier transform of $R(\mathbf{x}, \zeta)$ (three-dimensional in $2 \mathrm{D})$, will allow us to map $\hat{R}\left(\mathbf{k}, k_{\zeta}\right)$ to its angle gather equivalence, $\hat{R}(\mathbf{k}, \theta)$ using equation 2 . For filtering purposes, we use equation 2 to filter out the image corresponding to large $\theta$ (transmissions) or the gradient energy corresponding to small $\theta$ (reflections), and just sum the rest over $k_{\zeta}$ (the zero $\zeta$ imaging condition). As a result, there is no need to map to angle gathers. Of course, we will have to inverse Fourier transform the image back to space. Obviously, we also mute all energy laying in regions of $\hat{R}\left(\mathbf{k}, k_{\zeta}\right)$, where $\cos \frac{\theta}{2}>1$.

Relating equation 2 to the model update wavenumber demonstrates that $k_{\zeta}=\frac{\omega}{v}$, which allows us to directly control the frequency content scaled by the velocity in the update. In other words, $k_{\zeta}$ controls the update wavenumber scale. Thus, the model update can be written:

$$
\mathbf{k}=k_{\zeta} \cos \frac{\theta}{2} \mathbf{n}
$$

This allows for a full control of the model update wavelength regardless of frequencies.

For a constant velocity background the extension is not needed as the filtering is space independent. Thus, a $\zeta$ Fourier transform of equation 1 yields:

$$
\hat{R}\left(\mathbf{k}, k_{\zeta}\right)=R(\mathbf{k}) \delta\left(k_{\zeta}-2 \frac{\omega}{v}\right)
$$

which implies that $k_{\zeta}=2 \frac{\omega}{v}$, which can be used directly to filter $\hat{R}(\mathbf{k})$ using equation 3 with out the extension. As a result, the mapping equation becomes:

$$
|\mathbf{k}|=\frac{2 \omega}{v} \cos \frac{\theta}{2}
$$

Thus, the filtered gradient has the following form:

$$
R_{f}(\mathbf{x})=F^{-1} w(\mathbf{k}, v, \omega) F R(\mathbf{x}),
$$

where $F$ and $F^{-1}$ stands for the Fourier and inverse Fourier transform, respectively, in space. To mitigate low frequency imaging artifacts (Khalil et al., 2013):

$$
w(\mathbf{k})=\left\{\begin{array}{l}
1, \text { if } \cos \frac{\theta}{2}>\frac{v|\mathbf{k}|}{\omega} \\
0, \text { if } \cos \frac{\theta}{2} \leq \frac{v|\mathbf{k}|}{\omega}
\end{array},\right.
$$

for a given angle $\theta$.

To mitigate the nonlinear parts of the FWI gradient (Alkhalifah, 2015b):

$$
w(\mathbf{k})=\left\{\begin{array}{l}
0, \text { if } \cos \frac{\theta}{2}>\frac{v|\mathbf{k}|}{\omega} \\
1, \text { if } \cos \frac{\theta}{2} \leq \frac{v|\mathbf{k}|}{\omega}
\end{array} .\right.
$$

In both cases, the filtering will benefit from a tapering to reduce Gibb's phenomena effect.

\section{THE INTERPOLATION APPROACH}

Since the constant velocity filtering is considerably faster than relying on an axis extension, we can devise an approximation based on a reduced dimension and rely on interpolation. The reduced axis is given by the velocity magnitude. We, specifically, decimate the velocity and implement constant velocity scattering angle filtering, and use the actual velocity to interpolate between the constant velocity filtering. In this case, equation 6 has the following form:

$$
R_{i}(\mathbf{x})=F^{-1} w\left(\mathbf{k}, v_{i}, \omega\right) F R(\mathbf{x}),
$$

where $i$ is an index corresponding to a constant velocity between the minimum and maximum velocity value in our model, with $i=1 \ldots n$. If we rely only on the minimum and maximum velocity, $n=2$.

We can then interpolate the final image or gradient:

$$
R_{f}(\mathbf{x})=\sum_{i=1}^{n-1} a_{i}\left(\frac{v^{k}(\mathbf{x})-v_{i}^{k}}{v_{i+1}-v_{i}^{k}} R_{i+1}+\frac{v_{i+1}^{k}-v^{k}(\mathbf{x})}{v_{i+1}^{k}-v_{i}^{k}} R_{i}\right),
$$

where $k$ controls the velocity moment

$$
a_{i}=\left\{\begin{array}{l}
1, \text { if } v(\mathbf{x})>v_{i} \quad \& \quad v(\mathbf{x}) \leq v_{i} \\
0, \text { else }
\end{array} .\right.
$$

Higher-order interpolants, like splines, are possible.

Since each of the filters are positive definite as it amounts to a smoothing application. There linear combination given by the interpolation maintains the positive definite 
nature of the preconditioning, regardless of the velocity used in the interpolation. However, since the object is to maintain a smooth update. For a complex velocity model, like the Marmousi, the linear interpolation in the space domain may inject high wavenumbers as abrupt changes in the velocity model will be reflected in the interpolated gradient. To mitigate this problem, we use $v$ in equation eq: 17 that is exposed to the same smoothness. In conventional FWI implementation, we move slowly from high to low scattering angle starting from a smooth initial velocity model. Thus, this issue is less prominent.

\section{GRADIENTS UNDER FILTERING}

Though an efficient implementation of the filtering can be used for mitigating low frequency artifacts and improving the image resolution, we will focus here on it's use to filter the FWI gradient. The scattering angle filter can be applied directly to any adjoint state method based gradient, including second-order adjoint used for Hessian Calculation. In the examples below, we use gradients obtain from conventional FWI. We will first compare the approximate filter to the exact one.

For a linearly increasing velocity model shown in Figure 1(a), the gradient (sensitivity kernel) for a single source and receiver, and a frequency of $7 \mathrm{~Hz}$ is shown in Figure 1(b). The kernel in this example was computed using the ray-Born approximation, with the traveltime, as shown in Figure 1(a) (counter curves), calculated under the high-frequency asymptotic assumption. The gradients reflect the monochromatic nature of the wavefields used here, and we can clearly identify the low wavenumber wave path parts of the gradient, as well the scattering parts, given by the high wavenumbers. For this single frequency experiment, these parts are related directly to the scattering angle between the state and adjoint wavefields. Using a scattering angle filter that allows angles above 175 degrees to survive, we end up with the gradient shown in Figure 3(a). The filtering required the computation of an extended axis for $\zeta$, as shown in Figure 2. This increases the cost by $N \log N$ compared to a direct wavenumber filtering to the gradient, where $N$ is the number of samples of the extended axis, $\zeta$. In this example, I used 200 zeta samples at a sampling rate of $0.02 \mathrm{~km}$. Alternatively, if we apply constant velocity filters using the minimum and maximum velocity in this model $(1500$ and $4500 \mathrm{~km} / \mathrm{s}$, respectively) and interpolate between the two gradients using $k=2$ in equation 10, which effectively doubles the cost of a direct wavenumber filter, we get the gradient shown in Figure 3(b), which maybe considered as a reasonable approximation to Figure 3(a). The importance of the velocity dependency of the filter lies in trying to satisfy the scattering angle requirements of transmission components, which also depend on the velocity.

The Marmousi data Gradient

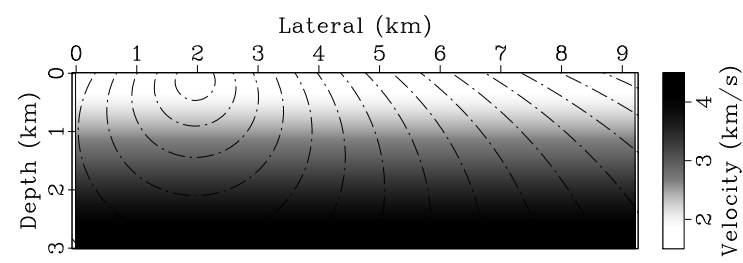

(a)

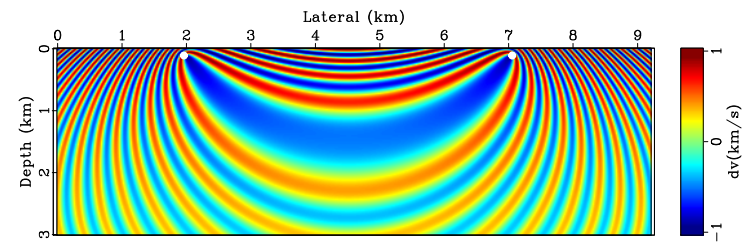

(b)

Figure 1: a) A linear velocity increasing model. The traveltime solution from a source located at the surface is given as a contour overlay. b) The sensitivity kernel for $7 \mathrm{~Hz}$ monochromatic wavefields for source and receiver locations given by the white dots.

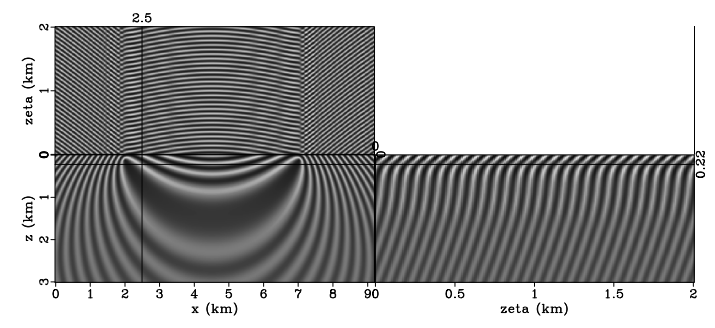

Figure 2: The $\zeta$ extended gradient based in equation 1.

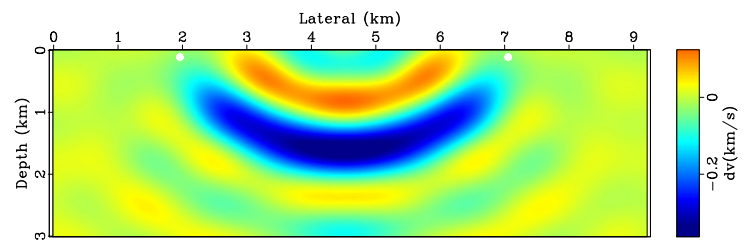

(a)

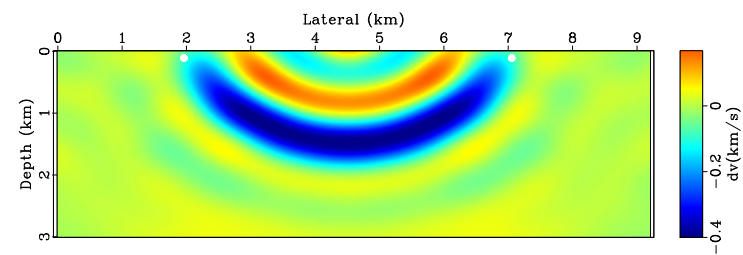

(b)

Figure 3: a) The scattering filtered gradient in Figure 1(b) using the extension. b) The scattering filtered gradient without the extension, using the interpolation. The scattering angle filter high pass cut off is 175 degrees. Also shown is the locations of the source and receiver for this kernel as white dots. . 
Now, we investigate the accuracy of the fast scattering angle filter on a gradient corresponding to the Mamrousi data set. Thus, using the Marmousi model (Figure 4(a)), I generate data for 64 shots covering the surface of the model using a Helmholtz solver for a frequency of $5 \mathrm{~Hz}$. I consider an initial velocity model given by a constant vertical gradient of $1 s^{-1}$, and a surface velocity of $1.5 \mathrm{~km} / \mathrm{s}$. Using the adjoint state method, I calculate the gradient, which is shown in Figure 4(b). The gradient looks like an inaccurate image of the Marmousi model as the velocity used is inaccurate. This gradient could be helpful only if the scattering prediction is within a half wavelength away from it's true scattering point at every point in the model. This tends usually not to be the case for the deeper parts.

Extending the gradient in Figure 4(b) in zeta provides us with an additional axis necessary to apply a scattering angle decomposition of the energy involved in calculating the gradient, as shown in Figure 4(c). Applying a scattering angle filter that passes only energy corresponding to scattering angles beyond 178 degrees provides the gradient in Figure 4(d). It is clearly smoother than the original gradient and free of the source location imprint. The extension, however, increased the cost of the gradient calculation by a considerable amount. Using the minimum and maximum velocity plus interpolation, we obtain the gradient shown in Figure 4(e). This approach is, off course, an approximation and thus, the two gradients are slightly different. However, the difference is reasonable, and I may argue that the gradient resulting from the approximate filtering looks more interesting as it shows sharper features up shallow and it looks smoother at depth, which is an objective of the scattering angle filtering. In the presentation, I will show the application of the efficient filter in FWI.

\section{CONCLUSIONS}

Scattering angle filters can help improve our migration image by mitigating low frequency transmission based noise, but more importantly, it can help full waveform inversion by producing gradients dominant by the transmission components. I develop an efficient approximate implementation of the scattering angle filter free of the extension axis, which was necessary to address the non stationary nature of the filter. The cost is synonymous to applying almost two or three stationary filters, and thus, the cost reduction is high. The resulting filtered gradient for FWI emphasis the long wavelength components when the scattering angle cut off is large, which is an objective of FWI in the early iterations.

\section{ACKNOWLEDGMENTS}

I thank KAUST for its support and the seismic wave analysis group for the many fruitful discussions I had with its members.

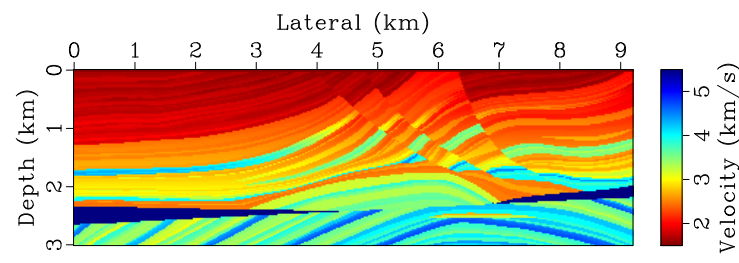

(a)

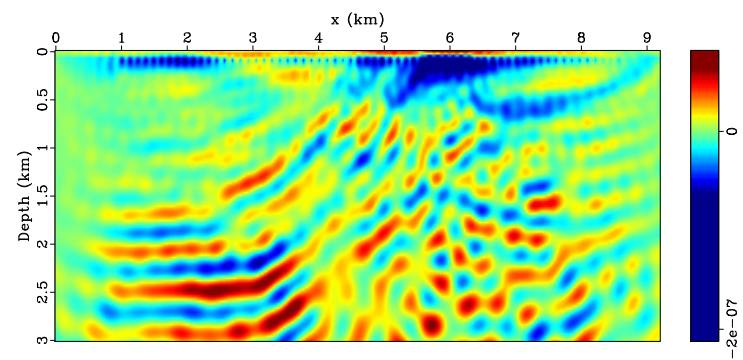

(b)

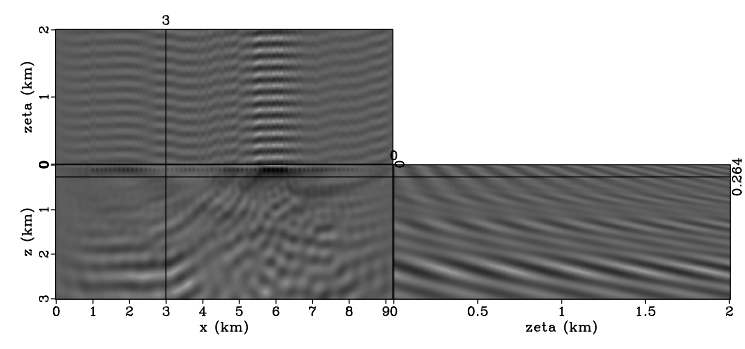

(c)

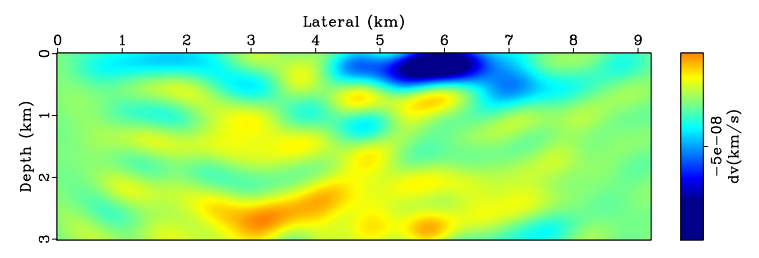

(d)

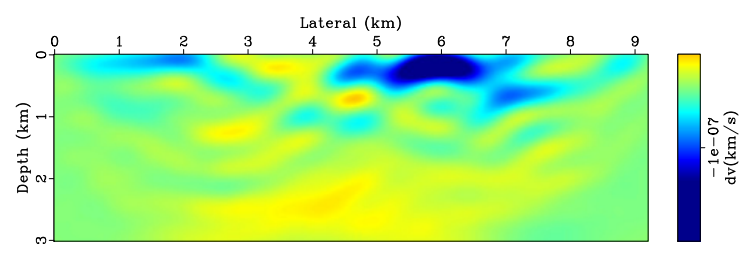

(e)

Figure 4: a) The Marmousi model. b) The gradient corresponding to a linear velocity increasing background model. c) The $\zeta$ extended version of the gradient. d) The scattering filtered gradient in Figure 4(b) using the extension. e) The scattering filtered gradient without the extension, using the interpolation. The scattering angle filter high pass cut off is 178 degrees. 


\section{EDITED REFERENCES}

Note: This reference list is a copyedited version of the reference list submitted by the author. Reference lists for the 2015 SEG Technical Program Expanded Abstracts have been copyedited so that references provided with the online metadata for each paper will achieve a high degree of linking to cited sources that appear on the Web.

\section{REFERENCES}

Alkhalifah, T., 2015a, Conditioning the full waveform inversion gradient to welcome anisotropy: Geophysics, 80, no. 3, R111-R122.

Alkhalifah, T., 2015b, Scattering-angle based filtering of the waveform inversion gradients: Geophysical Journal International, 200, no. 1, 363-373, http://dx.doi.org/10.1093/gji/ggu379.

Gazdag, J., and P. Sguazzero, 1984, Migration of seismic data by phase shift plus interpolation: Geophysics, 49, 124-131, http://dx.doi.org/10.1190/1.1441643.

Khalil, A., J. Sun, Y. Zhang, and G. Poole, 2013, RTM noise attenuation and image enhancement using time-shift gathers: 83rd Annual International Meeting, SEG, Expanded Abstracts, 3789-3793.

Sava, P. C., and S. Fomel, 2006, Time-shift imaging condition in seismic migration: Geophysics, 71, no. 6, S209-S217, http://dx.doi.org/10.1190/1.2338824.

Sirgue, L., and R. Pratt, 2004, Efficient waveform inversion and imaging: A strategy for selecting temporal frequencies: Geophysics, 69, 231-248, http://dx.doi.org/10.1190/1.1649391.

Yoon, K., K. J. Marfurt, and W. Starr, 2004, Challenges in reverse time migration: 74th Annual International Meeting, SEG, Expanded Abstracts, 1057-1060.

Zhang, Y., and J. Sun, 2008, Practical issues of reverse time migration: True-amplitude gathers, noise removal and harmonic-source encoding: 70th Conference \& Exhibition, EAGE, Extended Abstracts, F047, doi:10.3997/2214-4609.20147708. 